Issued by Sandia National Laboratories, operated for the United States Department of Energy by Sandia Corporation.

NOTICE: This report was prepared as an account of work sponsored by an agency of the United States Government. Neither the United States Government, nor any agency thereof, nor any of their employees, nor any of their contractors, subcontractors, or their employees, make any warranty, express or implied, or assume any legal liability or responsibility for the accuracy, completeness, or usefulness of any information, apparatus, product, or process disclosed, or represent that its use would not infringe privately owned rights. Reference herein to any specific commercial product, process, or service by trade name, trademark, manufacturer, or otherwise, does not necessarily constitute or imply its endorsement, recommendation, or favoring by the United States Government, any agency thereof, or any of their contractors or subcontractors. The views and opinions expressed herein do not necessarily state or reflect those of the United States Government, any agency thereof, or any of their contractors.

Printed in the United States of America. This report has been reproduced directly from the best available copy.

Available to DOE and DOE contractors from Office of Scientific and Technical Information

P.O. Box 62

Oak Ridge, TN 37831

Prices available from (703) 605-6000

Web site: http://www.ntis.gov/ordering.htm

Available to the public from

National Technical Information Service

U.S. Department of Commerce

5285 Port Royal Rd

Springfield, VA 22161

NTIS price codes

Printed copy: A03

Microfiche copy: A01

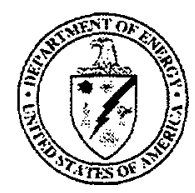




\section{DISCLAIMER}

Portions of this document may be illegible in electronic image products. Images are produced from the best available original document. 
SAND99-2587

Unlimited Release

Printed October 1999

\title{
Functional Requirements for SIERRA Version 1.0 Beta
}

\author{
Lee M. Taylor, H. Carter Edwards, and James R. Stewart \\ Advanced Software Environments \\ Sandia National Laboratories \\ P. O Box 5800 \\ Albuquerque, New Mexico 87185-0827
}

\begin{abstract}
This report contains a statement of the requirments for the SIERRA Version 1.0 Beta release.
\end{abstract}




\section{Acknowledgment}

The authors gratefully acknowledge the developer community within Sandia. These requirements came out of numerous discussions with the Presto, Adagio, Calore and Fuego application development teams. 


\section{Introduction}

The objective of the SIERRA framework is to provide a common software infrastructure for massively parallel computational mechanics applications. The SIERRA framework consolidates the mechanics-independent computational services required by a diverse set of mechanics applications into a shared framework. Consolidation of these computational services eliminates their redundant development and maintenance efforts and streamlines the coupling of independently developed computational mechanics capabilities into integrated multi-mechanics applications.

\subsection{Initial Focus on Finite Element Method (FEM) Applications}

The SIERRA framework will initially focus on the computational services required for applications that use the finite element method on unstructured meshes. These services include, but are not limited to, generalized parallel data structures, math kernels, interfaces for mechanics algorithms, and support for coupling mechanics capabilities developed using the SIERRA framework.

- Finite Element Services - this capability contains the basic core libraries of finite element types necessary to support the general purpose mechanics applications. In SIERRA this consists of a library of Master Elements which hold all the discrete calculus methods necessary for the applications (e.g. gradient operator, interpolation, numerical integration rules, etc.). This infrastructure also holds the Math Library -- a basic set of functionality which can be applied to tensors and vectors (e.g. rotate a tensor, calculate principal values of a tensor, etc.).

- Multi-mechanics Services - the capability to rapidly couple single physics applications into a multi-physics package. This capability is based on the solution transfer operators built into SIERRA as high level objects. These transfer objects are parallel in nature and highly scalable. The transfer algorithm can manage the transfer of a solution field from one mesh to a different mesh, each of which can have its own domain decomposition. The developer of the multi-physics package need not understand every detail of the single physics packages. The high level interface is couched in terms such as, "move this field from this mesh to that mesh."

- Adaptivity Services - the capability to divide and undivide elements based upon mechanics criteria. The interface to the mechanics developers is simply to mark an element to be divided, undivided, or left as is. The mechanics developer writes the error estimator and the algorithm for marking the elements for division/undivision. Also, the mechanics developer selects one of several possible prolongation/restriction operators (the rules for how to move data from parents to children, and back). Once an element is marked for division/undivision, SIERRA takes over and manages the data. The mechanics developer need not understand the details of this data management.

- Surface Mechanics Services - in SIERRA, we provide an abstraction of a surface and a set of interfaces for performing mechanics operations on the surfaces. Support for scalable parallel surface mechanics algorithms is key to the design of this service. In general, the surface of a model represents the skin of some underlying solid model (represented as a mesh). The domain decomposition of the underlying volume mesh does not represent a balanced decomposition of the discrete surface representation. SIERRA provides mechanisms for creating appropriate balanced decompositions of the surfaces independent of the underlying volume mesh. These secondary balanced decompositions of the surfaces are fundamental to obtaining scalable surface 
algorithms (e.g. contact searching). A major feature of the surface mechanics services is that the surface objects in SIERRA are cognizant of the data topology changes occurring under adaptivity and provide an automatic rebuilding of the discrete surface mesh as the underlying volume mesh is changing under the adaptivity criteria.

- Scalable Linear Equation Solver - SIERRA provides a common interface to linear solver services for scalable solution of sparse systems of equations on distributed and shared memory parallel architectures. SIERRA's interface to linear algebra packages is based upon a finite element view of the process. The underlying linear algebra representation of the assembled global element operators and right-hand-sides is not exposed to the mechanics application developer. This abstraction layer allows the interchange or selection of different linear algebra solvers and/or preconditioners without modifying the mechanics implementation.

- Parallel IO - provides a parallel IO infrastructure which is independent of the file format. SIERRA provides an abstraction layer between the underlying neutral file format and the mechanics application developer. This service provides the ability to write results or restart files in parallel to one or more neutral file formats. New neutral file formats can be added to the system and become immediately available to mechanics applications without modifying the applications code. The neutral file format is selectable at run time from the input file.

- Dynamic Load Balancing - provides a set of methods for moving mesh objects and their associated data from one processor to another. SIERRA will include a library of decomposition tools and methods which have been developed at Sandia to aid in this process.

\subsection{Evolving Framework}

The scope of the SIERRA framework is not limited to computational mechanics using the finite element method on unstructured meshes. It is planned for the SIERRA framework to continuously evolve from this initial focus to include "meshless" finite element methods, multiblock finite difference methods, particle methods, or other methods supporting computational mechanics. Furthermore, it is expected that the SIERRA framework will evolve to support the ever increasing complexity required by increasingly sophisticated mechanics applications.

The continuous evolution of the SIERRA framework is managed through the development of a conceptual framework that expresses the services provided by the SIERRA framework in rigorously defined mathematical abstractions. It is anticipated that this conceptual framework will provide for well-defined and efficient evolution of the SIERRA framework.

\subsection{Requirements Terminology}

\section{"Shall" and "Should"}

Each software requirement for the SIERRA framework are stated in a single sentence containing the word "shall." Occasionally a requirement-like statement is given with the word "should." These "should" statements typically focus on (vague) performance considerations that do not effect functionality of the SIERRA framework. 


\section{Programmatic Requirements}

Some requirements are listed under a subclassification of "Programmatic Requirements." These requirements do not address required functionality or performance of the framework, but reflect programmatic decisions that subsequently shape and guide the design and implementation of the SIERRA framework. 


\section{Computational Environment}

\subsection{Assumptions}

It is assumed that the target computational environment for applications developed using the SIERRA framework is massively parallel, distributed memory, and homogeneous.

The SIERRA framework is intended for applications computing on large meshes.

\subsection{Parallel Environment}

\subsubsection{Shall use the Single Program Multiple Data (SPMD) model.}

The SIERRA framework is developed based upon the data-parallel model, not the task-parallel model.

\subsubsection{Shall use the Message Passing Interface (MPI) for all communications.}

The MPI is the predominant standard for a message passing application programming interface (API). Message passing can be used for parallel processing in a shared memory or hybrid shared/ distributed memory environment.

\subsubsection{Shall define the parallel host by an application specified MPI communicator.}

This allows an application to partition its pool of processors into a SIERRA framework controlled set of processors and a disjoint set of processors that are "transparent" to the SIERRA framework.

2.2.4 Shall not be guaranteed to be thread-safe.

This "shall not" indicates that thread safety is not required and will not be considered.

\subsection{Warning/Error Handling}

2.3.1 Shall provide non-terminating message facility that coherently merges message text from all processors into a single output file.

\subsection{Computational Languages}

The SIERRA framework is developed using the $\mathrm{C}++, \mathrm{C}$, and FORTRAN77 computational languages. 


\subsubsection{Shall support interfacing with software components with $\mathrm{C}++, \mathrm{C}$, and FORTRAN77 interfaces.}

\subsection{Coupling with non-SIERRA Application Object Code}

An application may require the integration of object code that is not developed using the SIERRA framework.

Interface requirements imposed on external object code packages

- If the package has persistent state information then the package must supply restart input/output functions.

- The package must not violate memory bounds.

- The external linkage names of the package (e.g. function names) must be segregatable through a 1) name prefix or 2) modification of source code to alter the linkage names.

- Must allow SIERRA to be the "driver" code, i.e. SIERRA controls the application and invokes the external package.

- If the package performs parallel operations then it must use MPI and must accept an MPI communicator for its operations. 


\section{Mesh (Discretization) Services}

- Definition: A SIERRA Mesh is an application defined network of entities that represents the discretization of an application's domain space.

- Definition: An atomic entity within a SIERRA Mesh is a SIERRA Mesh Object.

- Definition: Each instance of a SIERRA Mesh resides within a SIERRA Region.

Note that a SIERRA Region may contain more that one SIERRA Mesh.

\subsection{Mesh Objects}

\subsubsection{Shall support relationships between any arbitrary pair of mesh objects.}

- Definition: A mesh object relation defines the relationship between a pair of mesh objects.

Example: A hexahedral finite element has relations with the eight nodes defining its vertices.

Example: A constraint between two or more mesh objects is itself a mesh object.

\subsubsection{Shall support relationship attributes of ordering and orientation.}

Example: A hexahedral finite element may have relations with the six mesh objects representing its faces. Each element-face relationship may denote a particular ordinal and orientation of the face with respect to the element.

\subsubsection{Shall support implementation of FEM mesh object topologies and FEM mesh objects.}

- Notation: $M_{D}^{d}$ is a "d-dimensional" FEM mesh object in a D-dimensional space where $d \leq D$.

FEM-Nodes are associated with points in space, a "zero dimensional" mesh object $M_{D}^{0}$.

FEM-Edges are associated with simple line segments in space, a "one dimensional" mesh object $M_{D}^{1}$ defined by two end-point nodes and zero or more interior nodes.

FEM-Faces are associated with simple facets in space, a "two dimensional" mesh object $M_{D}^{2}$ defined by at least three simply connected edges and at least three vertex nodes.

FEM-Elements associated with simple volumes in space, a "three dimensional" mesh object $M_{D}^{3}$ defined by a set of boundary faces and/or edges and/or vertex nodes.

An FEM mesh object topology defines the dimensionality and mesh object relationships. 


\subsubsection{Shall support FEM mesh object topological queries.}

For example, querying an element for the nodes it uses or querying an edge for the elements that use it.

\subsubsection{Shall support implementation of mesh object constraint topologies and constraints between mesh objects.}

A mesh object constraint topology defines a set of mesh object types that may have non-mesh relationships. For example, a face in contact with a node where the node is not part of that face's specification. These constraints are "mesh objects", and benefit from all support afforded mesh objects.

A constraint identifies the particular mesh objects, which satisfy the constraint topology, that are related via a constraint.

\subsection{Parallel Distributed Mesh}

3.2.1 In a parallel globally consistent SIERRA Region every mesh object of a particular type shall have a globally unique identifier.

\subsubsection{Shall distribute the mesh objects among processors.}

This implies that SIERRA will not automatically replicate all mesh objects on all processors. However, an application may define a parallel distribution that will cause all mesh objects to be replicated on all processors.

3.2.3 Each mesh object shall be owned by a single processor and reside on the owning processor.

\subsubsection{Shall support mesh object sharing among processors.}

Mesh objects may reside on more than one processor. The processor extent (e.g. sharing processors) of a given mesh object shall be available on each sharing processor, i.e. on each processor on which the mesh object resides.

3.2.5 If a given mesh object resides on more than one processor then each processor's copy of that mesh object shall be identified with the same globally unique identifier.

\subsubsection{Shall support identification of shared mesh object access permissions.}

For example, a mesh object residing on a processor may be identified as modifiable or read-only. Note that this requirement is for identification of access permissions, it does not address enforcement of access permissions. 
3.2.7 The owning processor of a mesh object shall always have permission to modify the mesh objects.

\subsubsection{Mesh object relations shall be supported on the local processor.}

Note that this requirement implies that interprocessor mesh object relationships, such as a particular mesh object residing on more than one processor, will be supported by a different mechanism.

\subsection{Subsets of Mesh Objects}

\subsubsection{Shall support specification of subsets of mesh objects.}

An initial list of subset specifications includes:

1. mesh object type (node, edge, face, element, constraint,...),

2. input subset specifications (element blocks, face sets, edge sets, node sets,...),

3. mesh object topology,

4. mechanics algorithms applied to mesh objects,

5. which mesh of a multi-mesh application (multilevel, multigrid,...),

6. parallel distribution (owned, shared, ghosted), and

7. intersections and unions of other subsets.

3.3.2 Shall manage all mesh objects of a SIERRA Region within SIERRA Rosters.

- Definition: A SIERRA Roster is a container for mesh objects that are of the same type.

3.3.3 Shall provide access to any application specified subset of mesh objects contained in a SIERRA Region.

An application will be able to efficiently iterate through a specified subset of mesh objects.

\subsubsection{Shall support application specification of arbitrary subsets of mesh objects.}

3.3.5 Shall support insertion/removal of mesh objects from application specified subsets of mesh objects.

\subsubsection{Shall support querying a mesh object for all of the mesh object subsets in which the} mesh object resides.

This querying capability is needed by the contact algorithm to determine the contact properties. Specifically the node and face that are in contact are queried for properties that are defined over an entire subset, e.g. a surface or element block. 
3.3.7 Given a mesh object's region, type, and global identifier shall provide at most an order $\log (\mathbf{N})$ time-complexity search of the on-processor roster for the mesh object, where $\mathbf{N}$ is the number of mesh objects in the roster.

3.3.8 Shall provide at most an order $\log (\mathrm{N})$ time-complexity insertion of a new mesh object into an on-processor roster, where $\mathbf{N}$ is the number of mesh objects in the roster.

3.3.9 Given a mesh object shall provide a constant-time-complexity deletion of that mesh object from an on-processor roster.

\subsection{Dynamic Updates to Mesh Objects}

\subsubsection{Shall support parallel-global synchronization of local updates to mesh objects.}

Local updates to a region's mesh may render mesh data structures inconsistent between processors. It would be highly inefficient to propagate individual updates to mesh object between processors as the updates occur. A parallel-global synchronization service that propagates multiple updates to mesh objects would be more efficient.

\subsubsection{Shall support globally consistent dynamic creation and deletion of mesh objects.}

Dynamic creation is the post-mesh-input creation of mesh objects, in support of adaptive refinement, constraint introduction, or other mesh-modifying algorithms. Dynamic deletion is in support of adaptive unrefinement, constraint removal, element erosion, or other mesh-modifying algorithms. Consistency may be achieved through global mesh update synchronization.

\subsubsection{Shall support communication of mesh objects among processors.}

Communication of mesh objects has two related applications: 1) create a copy of a mesh object on a different processor and 2) create a copy of a mesh object on a different processor, forward ownership of that mesh object to that processor, and delete the old copy of the mesh object.

Communication of mesh objects is needed to support dynamic load balancing, intra-Region operations such as contact mechanics, and inter-Region operations such a multi-mechanics transfers.

\subsection{Dynamic Generation of "Intermediate" of FEM Mesh Objects}

Given a consistent subset of FEM mesh objects, for example the faces and nodes of a surface, an application may need the edges within that surface to perform a given computation.

3.5.1 Given a consistent subset of FEM faces and nodes shall generate, upon request, the edges of that subset of faces. 


\section{Variable (Field) Services}

Mechanics computations are supported by variables or fields defined on specified subsets of mesh objects. Within this mechanics-specified subset each mesh object will be assigned values that are associated with each of the mechanics' variables.

\subsection{Variable Types}

4.1.1 Shall support predefined scalar, vector, and tensor types with real-valued coefficients.

\subsubsection{Shall support specification of the SIERRA-intrinsic real and integer types.}

Only one intrinsic type for real values is supported. This intrinsic real type is currently double precision (64bits).

\subsubsection{Shall support application defined arrays of intrinsic real and integer values.}

\subsubsection{Shall support application defined aggregates of named variables of other defined variable types.}

The variable types of the aggregate's member variables must be defined prior to the application's definition of the aggregate type. I.e., incomplete type definitions will not be supported.

- Definition: A named aggregate data type is termed a SIERRA Registrar. 


\subsection{Variable Classification and State Variables}

4.2.1 Variables shall be classified as either temporary, model, persistent, or state.

4.2.2 Temporary variables shall not be required for restart.

4.2.3 Model variables shall be initialized once, never modified, and required for restart.

4.2.4 Persistent variables are updated and required for restart.

4.2.5 State variables are updated, required for restart, and have values associated with each application defined state.

4.2.6 Each application shall specify which states of state variables are required for that application's restart output.

\subsection{Association of Variables with Mesh Objects}

4.3.1 Variable definitions shall be global information, i.e. shall not be associated with any particular instance of a mesh object.

4.3.2 Variables shall be associated with defined subsets of mesh objects.

4.3.3 Storage for values shall be managed for each variable associated with a mesh object.

The pairing of a variable with a mesh object, in an associated subset of mesh objects, defines storage for the values of that variable.

4.3.4 Storage shall not be present/allocated for variables that are not associated with mesh objects.

Given a variable and a mesh object, if the mesh object is not a member of the variable's specified subset of mesh objects then storage shall not be allocated for corresponding "non-existent" value.

4.3.5 Storage shall be directly accessible for each valid association of a variable and mesh object.

For example a pointer to the storage would be available. This pointer may be volatile in that any update to the mesh object may result in changes to the storage for variables associated with the mesh object. 


\subsubsection{Storage for a variable and a valid homogeneous subset of mesh objects should be maintained in a data structure that is most efficient for computations.}

This requirement is stated as a "should" rather than a "shall" because it is a performance requirement.

Given a homogeneous set of mesh objects, i.e. same type, connectivity, mechanics usage, etc., the storage for values associated with this homogeneous subset could be maintained in a single contiguous block of memory.

\subsection{Variable's Value Array Layouts}

\subsubsection{The value array of a variable shall be laid out, using the FORTRAN language notation, as 'array(nType,nCompLeft,nObjects,nCompRight)'.}

The dimensions of this array are:

1. nType, the number of scalar values in the variable's type (nType $=3$ for a Vector $3 \mathrm{~d}$ type),

2. nObjects, the number of mesh objects in the array, and

3. nCompLeft and nCompRight, the number of components of the variable type specified by the variable's data topology.

The storage for the value array shall be at least the product of the size of the intrinsic type, nType, nObjects, nCompLeft, and nCompRight.

\subsection{Variable Aliasing}

\subsubsection{Shall provide means to temporarily alias variable text names such that a given variable may be referenced by specified alias text names other than the originally declared text name.}

Some algorithms may expect a variable of a given text name. The variable to be passed to the algorithm may have been declared with a different text name. An aliasing capability would provide an application with greater flexibility in reusing algorithms. 


\subsection{Parallel Operations on Variable - Mesh Object Subset pairs}

4.6.1 Copy values from a source (domain) subset of mesh objects to compatible a destination (range) subset of mesh objects.

4.6.2 Assemble values within a subset of mesh objects that are shared between processors (i.e. the domain subset is identical to the range subset).

4.6.3 A successful parallel operation on variable - mesh object subset pairs shall yield consistent results on all processors and for identical invocations of the operation.

Identical invocations of an operation have the same host computer, number of processors, executable code, and input to the operation. This requirement is intended to insure that the SIERRA framework protects an application from the potential of non-deterministic message passing. 


\section{Mechanics Algorithm Support Services}

\subsection{Definitions}

- Definition: A mechanics algorithm is an operation applied to a subset of mesh objects and their variables' values.

Mechanics algorithms operate on a specified subset of mesh objects, e.g. an "element blocks", "surfaces", or "node lists". Mechanics algorithms can be grouped according to coupled and/or related operations, e.g. the coupled phases of contact mechanics or the suite of mathematical operations defined by a master element. Mechanics algorithms may be grouped in two ways: a sequence of algorithms with similar functionality, for example the enforcement of several boundary conditions, and an aggregation of algorithms, for example the separate initialize and execute phase of a mechanics calculation.

- Definition: The "root" aggregation of mechanics algorithms in an application is a Procedure.

- Definition: An application has one or more procedures that are sequenced by the Domain.

Mechanics algorithms may require supporting property data, control data, and/or functions to perform their operations. These supporting entities are associated with the application of a mechanics algorithm, they are not directly associated with any particular instance of a mesh object.

A mechanics algorithm may require one or more supporting mechanics algorithms, e.g. material models, friction coefficient models, or master element operations.

The scope of the mechanics algorithm support services is

1. specification of its required mesh object types and their variables,

2. specification of interdependencies,

3. managing the execution of mechanics algorithms.

\subsection{Aggregation of Mechanics Algorithms}

A "higher level" mechanics algorithm may utilize a coherent collection of mechanics algorithms, for example a collection of master-element operations. Such a collection defines an aggregation of mechanics algorithms. SIERRA currently supports several forms of aggregation for mechanics algorithms:

1. Element sections,

2. Element subsections,

3. Element materials,

4. Element blocks,

5. Surface uses,

6. Node uses (blocks), 
7. Constraint uses,

8. Regions,

9. Procedures, and

10. Domains.

These aggregations of mechanics algorithms represent specific instances of a more general abstraction for aggregate mechanics algorithms. A specification for this abstraction is under development; however, requirements for aggregates of mechanics algorithms are defined.

\subsubsection{Shall support extensibility of the types of aggregate mechanics algorithms.}

The previously noted types of aggregate mechanics algorithms may not be sufficient. SIERRA should support a straight-forward mechanism for extending this list.

\subsubsection{Shall manage a dynamic set of aggregated mechanics algorithms.}

There will be more than one instance of a type of aggregate mechanics algorithm; for example more than one master element or surface use. SIERRA will manage these multiple instances of aggregate mechanics algorithms.

\subsubsection{Shall manage the interdependencies of mechanics algorithms.}

The interdependencies of mechanics algorithms should be managed, for example, tracking which master element is used when an element algorithm applies a master element operation.

\subsubsection{Shall manage the interface specifications for mechanics algorithms.}

A particular type of aggregate mechanics algorithm may have a defined interface; for example the master element interface.

\subsubsection{Shall maintain parallel-global consistency of aggregate mechanics algorithms.}

All information (dependencies and data) associated with an aggregate mechanics algorithm is consistent across all processors.

\subsubsection{The types of aggregate mechanics algorithms shall have unique text-name identifiers.}

For example, the type-name of a surface use.

5.2.7 Shall support querying of an aggregate mechanics algorithm for its type(s).

For example, query a "generic" surface use for its specific type. 


\subsubsection{Shall support identification of related of mechanics algorithms as a group that may be accessed or processed as a group.}

The surface use 'type', which corresponds to all surfaces uses of a particular derived class type, is a specific example of a grouping of related mechanics algorithms.

Another example is the execution, with a single method call, of all element worksets of a given mechanics name that reside in any element block of a region.

5.2.9 Instances of aggregate mechanics algorithms shall have a unique text-name identifier.

Each aggregate mechanics algorithm is given a unique identifier, for example the name of an element block.

\subsection{Function Services}

- Definition: A function is a single operation and its supporting data.

\subsubsection{A function shall not operate directly upon mesh objects.}

A mechanics algorithm may apply a function to the variable values of a mesh object. However, the function's interface uses values not the mesh object.

\subsubsection{Shall manage functions and their supporting data.}

\subsubsection{Shall maintain parallel-global consistency of function modules.}

\subsubsection{Shall provide an initial extensible set of mathematical functions.}

1. Forward and backward rotation of vectors

2. Magnitude of a vector

3. Forward and backward rotation of symmetric tensors

4. Creation of direction cosine matrices from a quaternion

5. Creation of a quaternion from a direction cosine matrix

6. Calculation of principal values and directions of a symmetric tensor

7. Additive decomposition of a full tensor.

8. Polar decomposition of a deformation gradient tensor (full tensor).

9. Solution of a set of $\mathrm{NxN}$ equations at a point 


\subsection{Property Data and Control Data Services}

\subsubsection{Shall manage property and control data for an aggregated mechanics algorithms, using the SIERRA Framework Variable Services for consistency.}

Procedures, regions, element blocks and other aggregate mechanics algorithms may have data that is associated with an instance of the entire aggregate and not with any particular mesh object. For example the time step of a procedure.

\subsection{Master Element Services}

\subsubsection{Shall define an interface for Element Topology.}

The element topology module defines the shape, number of nodes, number of edges, etc.

\subsubsection{Shall define an (initial) interface for interpolation of basis functions that are continuous across element boundaries.}

Currently SIERRA supports Nodal Interpolation, where nodes may be placed at element vertices, on element edges, and on element faces. This functionality should be extended to include basis functions associated with edges and faces, as opposed to nodes on edges and faces.

\subsubsection{Shall define an interface for interpolation of element basis functions that are discontinuous across element boundaries.}

Element Interpolation - this class holds methods for interpolating element variables over the domain of the element. This does not mean that it provides an interpolation of the Gauss point variables. Rather it provides a mechanism for defining a field based on some set of element variables. The simplest and most common case of an element interpolation is a constant field --- obviously a trivial and not very interesting case. A more interesting case is a bilinear interpolation of some element field (say pressure) where the field is defined by a set of coefficients $p=a_{0}+a_{1} x+a_{2} y+a_{3} z$ where the four coefficients $\left(a_{0}, a_{1}, a_{2}, a_{3}\right)$ are element variables used to define the element field. These coefficients may actually be given to the linear equation solver as part of the global solution of degrees of freedom by the finite element method.

\subsubsection{Shall define an interface for Element Integration.}

An element integration mechanics module implements the methods that define the element integration rule (e.g. Gauss $2 \times 2 \times 2$ quadrature rule).

5.5.5 Shall define an interface for Master Element mechanics that consists of the aggregation of Element Topology, Element Nodal Interpolation, Element 


\section{Interpolation, Element Integration, Element Prolongation, and Element Restriction components.}

Provide a virtual base class hierarchy for the master element properties and discrete calculus operations. The master element base class hierarch defines a flexible and extensible interface for element technology. The master element class hierarchy is a set of lightweight base classes composed of its individual constituent classes (see below). Its "reason for existence" is to provide a consistent interface to element calculations. Individual derived classes can mix and match from the constituent classes. For example, suppose we have completed the implementation of the uniform gradient, eight node hex element. This implies that we have implemented the six constituent classes listed below. Now, to implement the numerically integrated (Gauss $2 \times 2 \times 2$ ) eight node hex element, we need only implement the single constituent class for Gauss quadrature (element integration) and we leverage the other 5 constituent classes.

\subsubsection{Shall provide an initial set of Master Elements.}

The following list contains the most common element formulations which will be needed by many if not most of the applications which are build upon the SIERRA framework.

1. Uniform Gradient Hex 8, 3D

2. $2 \times 2 \times 2$ Gauss Quadrature Hex $8,3 \mathrm{D}$

3. Uniform Gradient Quad 4, 3D

4. 2×2 Gauss Quadrature Quad 4, 3D

5. Uniform Gradient Quad 4, 2D

6. 2x2 Gauss Quadrature Quad 4, 2D

7. Tet $4,3 \mathrm{D}$

8. Tri 3,3D

9. Tri $3,2 \mathrm{D}$

10. Line $2,3 \mathrm{D}$

11. Line $2,2 \mathrm{D}$

12. $2 \times 2 \times 2$ Gauss Quadrature, Hex 20, 3D

13. 2x2 Gauss Quadrature, Quad 8, 3D

14. 2x2 Gauss Quadrature, Quad 8, 2D

15. Control Volume, Hex $8,3 \mathrm{D}$

16. Control Surface, Quad 4, 3D

\subsection{Section-Subsection-Material Services}

Complex, general purpose, models require a disparate collection of element formulations and material models. Typically the element formulations require sub-models which represent numeri- 
cal approximations to the true geometric representation of the model. An example of this arises in the formulation of a layered three dimensional shell element. The "element" might be a four node quadrilateral in three space. Hence, the element has a reduced topological dimensionality (from the $3 \mathrm{~d}$ solid to a $2 \mathrm{~d}$ shell). The through thickness behavior of such an element is generally represented by some type of "section" model in which the membrane and bending behavior is captured via a through-thickness integration rule which produces an element stress resultant and an element moment resultant. The section model might be broken down into sub-models as well (we refer to these as subsections). Why would we introduce a subsection? Consider the case where the shell element is representing a layered composite where each layer has different material properties and different orthotropic orientations. In such a case, the "section model" might have an orientation for the element while the "subsection model" might have an orientation of each layer with respect to the section orientation. Finally, the subsection model holds a set of material points (usually located at some material integration point locations through the thickness of the layer). For each of these material points, the subsection would invoke a particular material model appropriate to the physical material represented by the layer. 
5.6.1 Shall provide a general mechanism for the element mechanics algorithm to invoke a section mechanics algorithm.

5.6.2 Shall provide section mechanics algorithms with access to element, subsection and material variables' values.

5.6.3 Shall provide application defined section properties for a section mechanics algorithm.

5.6.4 Shall not provide section variables.

5.6.5 Shall provide a general mechanism for section mechanics algorithm to invoke a subsection mechanics algorithm for each subsection defined within the section.

5.6.6 Shall provide subsection mechanics algorithms with access to element, subsection and material variables' values.

5.6.7 Shall provide application defined subsection properties for a subsection mechanics algorithm.

5.6.8 Shall provide subsection-instance within an element variables.

5.6.9 Shall provide a general mechanism for invoking a material mechanics algorithm defined for the subsection.

5.6.10 Shall provide application defined material properties for a material mechanics algorithm.

5.6.11 Shall provide material mechanics algorithms with access to element, subsection and material variables' values.

5.6.12 Shall provide a general variable aliasing facility such that element, section, subsection, and material mechanics algorithms can internally rename variables.

\subsection{Workset Services}

5.7.1 Shall provide mechanics algorithms with the variable values of mesh objects in contiguous arrays.

5.7.2 Should aggregate contiguous arrays into blocks of memory sized for the host computer's cache.

This requirement is stated as a "should" rather than a "shall" because it is a performance require- 
ment.

\subsubsection{Shall support the application's specification of mesh-stencil for the workset.}

- Definition: An mesh-stencil defines a set of topologically connected mesh objects.

- Notation: \{primary-mesh-object: $\{$ connected-mesh-object $\} *\}$ The leading or primary entry in a mesh-stencil identifies the type of mesh objects that are iterated. The remaining entries identify the mesh objects topologically connected to the primary mesh object that are also processed. Examples of mesh-stencils include:

1. \{element: element-nodes\}

2. \{element: element-edges, element-nodes

3. \{face: face-edges, face-nodes $\}$

4. \{face: face-element, face-element-nodes\}

5. \{element: element-nodes, element-face-element, element-face-element-nodes\}

\subsubsection{Shall provide gather, scatter, and assemble operations to move variable values specified by a stencil and variable selection from non-contiguous storage to contiguous storage.}

A mechanics workset algorithm using an \{element, element-node\} mesh-stencil requires that the element-node variable values appear in a contiguous array, 'array(nType,nNodesPerElem,nElements)'. The variable values associated with an element's nodes must be gathered from non-contiguous memory into contiguous memory with the desired array layout.

\subsection{Element Block Mechanics Services}

- Definition: An element block:

a. Is a homogeneous collection of mesh object elements that defines a physical extent. The element blocks partition the set of elements in the Region.

This portion of the definition represents a subsetting mechanism. The element block defines a subset of the elements in the model.

b. Is the aggregation of one or more master element types and application defined mechanics algorithms that operate on elements.

This portion of the definition represents a "usage" construct. The element block defines a set of mechanics that can be performed on the subset.

An element block represents a homogeneous set of mesh objects have the same topology, mechanics, and variables. 
5.8.1 The family of subsets of elements defined by an application's element blocks shall be disjoint, i.e. a given element shall belong to exactly one element block.

An element can only belong to a single element block, but the nodes that define an element can be used to define elements in other element blocks. Elements belong to element blocks but nodes do not.

5.8.2 An element block shall be one subset of elements which are homogeneous with respect to element properties.

Examples of element properties are their master element(s) or material properties.

5.8.3 Conflicting assignments of elements to element blocks shall be resolved at the point of assignment, i.e. the conflict shall not be propagated into the data structure.

\subsubsection{Shall support specifications of groups of element blocks.}

A flat element block partitioning of a Region may not be sufficient. An application may require substructuring the Region via groups of element blocks.

\subsection{Mechanics Services for Arbitrary Subsets of Mesh Objects}

- Definition: A face-set is a collection of mesh object faces (or facets) that defines a physical extent.

- Definition: A edge-set is a collection of mesh object edges that defines a physical extent.

- Definition: A node-set is a collection of mesh object nodes.

- Definition: A surface use is the aggregation of one or more mechanics algorithms that operate on the faces (along with any defined face mesh-stencil), edges, and/or nodes of one or more surfaces.

- Definition: A line use is the aggregation of one or more mechanics algorithms that operate on the edges (along with any defined edge mesh-stencil) and/or nodes of one or more lines.

- Definition: A node use is the aggregation of one or more mechanics algorithms that operate on the nodes (along with any defined node mesh-stencil) of one or more node sets. 


\subsubsection{Shall support surface-uses.}

\subsubsection{Shall support edge-uses.}

\subsubsection{Shall support node-uses.}

\subsubsection{Shall support aggregation of mechanics algorithms applied to an arbitrary subset of mesh objects.}

\subsubsection{A face, edge, or node shall be associated with zero or more surface uses.}

\subsubsection{An edge or node shall be associated with zero or more edge uses.}

\subsubsection{An node shall be associated with zero or more node uses.}

\subsection{Constraint Mechanics Services}

Provide an abstraction of a constraint object handler. Examples of constraint objects are: single point constraints (i.e. boundary conditions), multipoint constraints (e.g. a rigid link), and contact constraints. Inherent in the abstraction must be the concept of the different types of constraints and the concept that some constraint types have precedence over others. Consider a surface which is part of a model which contains h-adaptivity. It will be quite common under this circumstance for a node on the surface to participate in a boundary condition, a multipoint constraint, and a contact condition simultaneously. The constraint handler object should provide a high level API for the mechanics developer to "teach" it about the constraints. This API should be formulated in terms of a set of phases in much the same way as the API for the Finite Element Interface to linear algebra services. In fact, the constraint handler is just another case of a linear algebra service. There are a few key differences. The constraint handler must have the capability to handle both implicit and explicit constraint requests (as described below). Also, the constraint handler must understand the hierarchy of constraints (i.e. precedence order). This hierarchy must be embedded into the constraint objects themselves and represents a set of heuristics which must be defined by the applications developer.

\section{Explicit Constraints}

Explicit codes typically enforce constraints in the form of a predictor corrector algorithm. The mechanics algorithm predicts the kinematics at the end of the next increment as if there were no constraints. Then, a set of constraint equations is formulated (for only the nodes that participate in constraints) that will correct the kinematics so that it is in compliance with the constraints.

The constraint handler object must assemble the set of constraints for a time increment and solve for the resulting corrections. The assembled set of equations is in general not sparse and is nonsquare. The constraint handler object will be required to modify the set of constraints to enforce the semantics of precedence and to factor the set of degrees of freedom into dependent and inde- 
pendent degrees of freedom. There is a wealth of linear algebra theory to be exploited to solve for this set of over constrained equations.

\section{Implicit Constraints}

Implicit finite element codes typically give the constraints to the linear algebra package to solve simultaneously with the global system of equations $(\mathrm{Ax}=\mathrm{B})$. The finite element interface to linear algebra services has a feature built into the API to achieve this. However, we still face the difficulty of dealing with the concept of conflicting constraints described above. The constraint handler object will be required to modify the set of constraints to enforce the semantics of precedence. It will then have the responsibility for handing the constraint equations to the FEI.

\subsubsection{Shall support application defined constraint topologies.}

A constraint topology defines the set of mesh objects that are constrained to one another.

\subsubsection{Shall support constraints with heterogeneous topologies.}

\subsubsection{Shall support aggregated constraint algorithms.}

\subsubsection{Constraints shall be managed within a SIERRA Roster.}

\subsubsection{Shall support precedence of constraint algorithms.}

5.10.6 Shall support dynamic addition and deletion of constraints. 


\section{Dynamic Parallel Decomposition / Load Balancing}

\subsection{Parallel Partitioning Specifications}

- Definition: A SIERRA parallel partitioning specification associates one or more processors with each mesh object in specified subset of mesh objects.

\subsubsection{Shall support creation and interrogation of parallel partitioning specifications.}

\subsection{Secondary (Auxiliary) Regions}

A secondary or auxiliary region is a region that

1. is an image of another primary region,

2. contains copies of a specified subset of mesh objects from the primary region,

3. has a different parallel decomposition of mesh objects than the primary region, and

4. is created for some computation and then discarded upon completion of that computation.

An example of where this capability would be used is in a contact algorithm. First, we could cast the face roster into a secondary decomposition using an RCB method. Then, we could do a proximity check which would return a (variable length) list of nodes for each face that are within the capture box of the face. We could then cull this list down to only those nodes which are actual contact candidates. Now, this resultant list would most likely be unbalanced in the existing secondary decomposition. However, we could then create another secondary geometric partitioning object based on the culled down list. Using this we would cast the culled down list into the new secondary decomposition.

\subsubsection{Shall support dynamic creation of secondary regions.}

\subsubsection{Shall support mesh object ghosting for secondary regions.}

Each processor participating in a secondary region may require copies of mesh object in addition to those mesh objects required to capture the interfaces of a decomposed mesh. For example, an interface layer of elements may resided on more than one processor. 
6.2.3 Input, restart, and output operations shall not be supported on secondary regions.

6.2.4 Shall support specification of variables in the Secondary Region independent of the specification of variables in the Primary Region.

6.2.5 Shall support specification of mechanics algorithms applied to the secondary region independent of the specification of mechanics algorithms applied to the primary region.

6.2.6 Shall support creation of copies of a specified subset of mesh objects from the primary region to the secondary region with a parallel partitioning specification.

6.2.7 Shall support copying variables's values between primary region variables and secondary region variables.

6.2.8 Shall support creation of secondary regions from other secondary regions.

6.2.9 Shall support connection between "chained" secondary regions.

Consider primary region $\mathrm{A}$, secondary region $\mathrm{B}$ created from primary region $\mathrm{A}$, and secondary region $C$ created from secondary region $B$. The connection between $A$ and $B$ and between $B$ and $\mathrm{C}$ are known from the creation of secondary region $\mathrm{B}$ and $\mathrm{C}$. The connection between primary region $A$ and secondary region $C$ must be obtainable. 


\section{Intra-Mechanics and Inter-Mechanics Transfer Services}

Transfer services transfer one or more application specified variable values from a subset of mesh objects in a sending region to application specified variables of a subset of mesh objects in a receiving region.

\subsection{Examples of Transfers}

\section{Nodal to Nodal Transfer}

Provide a method to transfer a nodal variable in the sending mesh to a nodal variable in the receiving mesh. This represents the classical "nodal interpolation" case.

\section{Nodal to Element Transfer}

Provide a method to transfer a nodal variable in the sending mesh to an element variable in the receiving mesh. Most of this requirement is fulfilled by the Nodal to Nodal transfer. Once the field is defined on the nodes of the receiving mesh, the variables can be transferred to the element integration stations using the interpolation methods defined upon the master element base classes.

\section{Element to Nodal Transfer}

Provide a method to transfer an element variable in the sending mesh to a nodal variable in the receiving mesh. This will require an extrapolation method for extrapolating the element variables to the nodes. Once the element variables are extrapolated to the nodes, they can be transferred to the receiving regions mesh using the nodal transfer methods.

\section{Element to Element Transfer}

Provide a method to transfer an element variable in the sending mesh to a element variable in the receiving mesh. This will require an extrapolation method for extrapolating the element variables to the nodes. Once the element variables are extrapolated to the nodes, they can be transfer to the receiving regions mesh using the nodal transfer methods and then using the interpolation methods on the receiving regions master elements.

\subsection{Same-Mesh Transfers}

\subsubsection{Shall support copying of selected mesh object variables' values between subsets of mesh objects within two same-mesh regions where the mesh objects within the subsets have identical identifiers.}

Same-mesh transfers should bypass interpolation or extrapolation and simply copy variable values. 
7.2.2 Shall support creation of constraint objects in a destination region as copies of constraint objects in a source region, given that the destination region 1) is the same mesh modulo the constraint objects and 2) does not currently have constraint objects.

\subsection{Different Mesh Geometry based Transfers}

7.3.1 Shall support interpolation of selected mesh object variables' values between specified subsets of mesh objects within two different mesh regions.

7.3.2 Shall provide on-processor geometric proximity geometric-search between specified subsets of mesh objects.

Geometric searching: A search method shall be provided for determining within which mesh object in the "searched mesh" a mesh object in the "searching mesh" is found. This represents a geometric based search such as used in a mesh to mesh transfer algorithm.

\subsubsection{The on-processor geometric search method shall be exhaustive and $\mathrm{O}(\mathrm{NlogN})$.}

\subsection{Parallel Geometric Search between Two Region Meshes}

Two region meshes or subsets of region meshes may be given a geometrically compatible parallel partitioning by creating secondary regions with the same geometric-based decomposition among processors.

\subsubsection{Shall determine how to geometrically partition specified subsets of two Region's mesh objects across processors to facilitate scalable geometric search.}

Satisfied via parallel geometric partitioning algorithm such as recursive-coordinate-bisection.

The partitioning of three dimensional space for a secondary decomposition should be independent of the construction of a distributed object using that partitioning. For example, the RCB algorithm partitions the three dimensional space by making a series of "cuts" where half of the objects are on one side of the cut and half on the other side of the cut. Then each cut piece is "cut" again. This goes on recursively until all the processors have an equal number of objects assigned. Usually the objects used to build such a decomposition are the finite element nodes. So, the partitioning is just a data structure consisting of a "tree" of cuts which describe how objects should be assigned to processors.

The reason we want to factor the geometric description of the secondary decomposition from the actual construction of a secondary decomposition of an actual distributed object using the cuts is that we may want to "push" more than one distributed object through this partitioning. In fact, we almost always push more than one object through. In the transfer objects, we create an RCB partitioning based upon the geometry of both the sending and receiving mesh. We then construct secondary decompositions of both the sending and receiving mesh using this common RCB 
partitioning. Hence we have mapped both the sending and receiving regions onto a common secondary decomposition.

7.4.2 If requested, the geometric partitioning shall balance the specified subsets of mesh objects among processors.

7.4.3 Shall support complete coverage with mesh objects of the space assigned to a processor by the geometric-based partitioning. 


\section{Linear Equation Solver Services}

\subsection{Assumptions}

Typically, linear algebra packages are formulated purely in terms of solving a set sparse set of $\mathrm{NxN}$ equations in the form $\mathrm{Ax}=\mathrm{B}$. These packages are usually written in terms of $\mathrm{N}$ degrees of freedom and some parallel distributed sparse matrix storage format that is optimized for the iterative solution method.

\subsection{Programmatic Requirements}

8.2.1 The SIERRA framework shall interface with a linear equation solver package through the application programming interface (API) named the Finite Element Interface (FEI).

The FEI is developed and supported by Sandia-Livermore. It defines services to create, fill, execute, and query results from parallel distributed sparse linear equations. The FEI services are FEM-centric; the interfaces are in the FEM semantics of elements, nodes, fields, boundary conditions, and constraints.

\subsection{Requirements for the FEI}

8.3.1 Shall support iterative solution of sparse linear systems of equations.

8.3.2 Shall support re-use of global linear system sparsity patterns.

8.3.3 Shall support fill and linear sum of multiple LHS and RHS contributions with identical sparsity patterns.

\subsection{Solver Options}

8.4.1 Shall support specification of FEI / solver package solution objectives.

Example: Single RHS, multiple RHS, eigenvalues,...

8.4.2 Shall support specification of FEI / solver package solution methods and preconditioners.

8.4.3 Shall support specification of FEI / solver package solution method controls.

Example: the iterative solution method stopping conditions. 


\subsection{Initialization Phase Services / Interface}

Initialization of an FEI linear system consists of describing the fields, nodes, elements, element matrices, element connectivities, constraint equations, and other FEM attributes.

8.5.1 Shall support specification of the subset of variables associated with the linear system of equations.

8.5.2 Shall support specification of the mechanics algorithms that contribute to the linear system of equations and the type of contribution.

A mechanics module may contribute element matrices or right-hand-side vectors, boundary conditions, constraints, etc.

8.5.3 Shall support specification of multiple left-hand-side (LHS) and right-hand-side (RHS) contributions to the linear system of equations.

This requirement supports the ability to perform scaled summation of multiple LHS / RHS.

8.5.4 Shall process variable, mechanics algorithm, and multiple LHS/RHS specifications along with internal region information to automatically map SIERRA data structure information onto the FEI initialization interface.

Note that this include initialization of the FEI required parallel distribution information.

\subsection{Fill / Assembly Phase Services / Interface}

The initialization procedure defines the structure and storage requirements for the parallel distributed linear system of equations. Once initialized LHS and RHS coefficients must be generated and filled into the linear system of equations.

8.6.1 Shall accept LHS and RHS partial sum contributions from specified mechanics algorithms.

8.6.2 Shall accept essential, natural, and mixed boundary conditions from specified mechanics algorithms.

Supported via the Finite Element Interface to linear solvers (FEI) definition of boundary conditions via the "alpha-beta-gamma" paradigm.

8.6.3 Shall accept strictly-enforced constraints.

These constraints are enforced within the FEI via Lagrange multipliers. 
8.6.4 Shall accept penalty-enforced constraints.

\subsection{Solution Phase Services}

8.7.1 Shall initiate solution of the linear system of equations.

8.7.2 Shall transfer the linear system of equations solution into the specified variable values. 


\section{Global Data Services}

Global data is classified as either global application data or global environment data. Global application data consists of options for mechanics algorithms, region values, and other information that can be specified in the absence of any instance of a mesh object. Global environment data includes information such as the number of processors or current working directory for output.

\subsection{Available Global Environment Data}

9.1.1 Shall provide parallel environment parameters of the number of processors and the local processor identifier.

9.1.2 Shall provide an execution phase parameter denoting at least the construction, initialization, restart, execution, normal termination, or error termination phases of an application.

\subsection{Management of Global Application Data}

9.2.1 All global application data shall be consistently duplicated on all processors.

9.2.2 Each item of global application data shall be identifiable as either constant or nonconstant.

9.2.3 Global application data that specifies which/how mechanics algorithms will be used within a given execution of an application shall be constant.

This implies that the algorithm interdependencies may not be changed after initialization. 


\subsection{Input of Global Application Data}

9.3.1 Global application data shall be input on the "root" processor and broadcast to all other processors.

9.3.2 Global application data shall be input for initialization and restart.

\subsection{Output of Global Application Data}

\subsection{Parsing of Input Leading to Global Application Data}

\subsubsection{Shall provide a consistent input syntax for all applications using the SIERRA} framework.

9.5.2 Shall support verification of consistency of input with the application's mechanics algorithms.

9.5.3 Input consistency verification shall be available "off-line" from the parallel execution, e.g. on a single processor of a local workstation.

\subsection{Pre-existing Design Decisions in regard to Parsing of Input}

Several design decisions were made for parsing application input. These design decisions are currently "irrevocable" and are therefore documented in the SIERRA software requirements.

- Shall have a flat textural representation of the high level objects in any SIERRA application, referred to as the Mechanics Definition $\underline{L}$ anguage (MDL).

- The MDL shall be defined and used to fulfill input global application data input requirements.

- Shall have an independent parser executable that parses input commands for any and all application executables built on the SIERRA framework, generates the required MDL, automatically determines which application executable is needed to satisfy the input, verifies the input, and upon request launches the executable on a specified host computer.

- This parser is developed and supported by an independent contractor.

- Shall provide an independent parser as a separate serial executable.

- The Parser shall read an user input specification and write an MDL specification.

- The Parser shall provide model verification.

- The Parser shall identify the proper application to be run from the input specification and provide launch services for the proper executable associated with that executable. 


\section{Bulk Data Input/Output Services}

Bulk data is the information associated with specific instances of mesh objects. Bulk data is often coupled to or dependent upon global application data; therefore, input/output of bulk data may be coupled to input/output of global application data.

\subsection{Input}

10.1.1 Shall support, upon request, input of bulk mesh-data.

Bulk mesh-data specifies the mesh objects and their relationships.

10.1.2 Shall support, upon request, input of all bulk numeric-data into mesh data structures.

Bulk numeric-data are the numerical values associated with variables of mesh objects. 
10.1.3 Shall not load bulk data unless specifically requested.

10.1.4 Shall provide globally unique identifiers for all mesh objects input to particular instance of a given region.

10.1.5 Shall identify the topology of input mesh objects.

\subsection{Output}

10.2.1 Shall output bulk mesh-data.

10.2.2 Shall output bulk numerical-data.

10.2.3 Shall provide nodal output for any or all registered nodal variables.

10.2.4 Shall provide element output for any or all registered element variables.

10.2.5 Selection of which output variables shall be defined at runtime by the analyst.

10.2.6 Shall provide element output for any or all registered element-subsection variables.

10.2.7 Shall provide element output for any or all registered element-subsection-material variables.

10.2.8 Shall support output-triggered execution of specified mechanics algorithms to compute derived data for output.

10.2.9 Shall support output of specified global application data.

10.2.10 Shall provide the capability for an application to define named algorithms for deriving an output variable from a registered nodal variable.

10.2.11 Shall provide the capability for an application to define named algorithms for deriving an output variable from registered variables within the element/subsection/ material registrar hierarchy.

10.2.12 Shall not provide the capability for an application to define named algorithms for deriving an output variable from registered variables within a control data object.

10.2.13 Selection of nodal output variables shall be defined at runtime by the analyst. 
10.2.14 Selection of element/subsection/material output variables shall be defined at runtime by the analyst.

10.2.15 Selection of global output variables shall be defined at runtime by the analyst.

\subsection{Restart}

10.3.1 Shall use the bulk data input/output services.

10.3.2 Shall support application specification of a fixed number of restart frames.

10.3.3 Shall support application specification of all restart frames.

10.3.4 Shall support restart output upon request.

10.3.5 Shall support restart output on a predefined schedule.

10.3.6 Shall support a single output file format for restart output.

\subsection{Multiple File Formats}

10.4.1 Shall be extensible for new file formats transparently to all mechanics algorithms.

Provide an abstraction for IO services which allows the mechanics developer to select such concepts as neutral file format and parallel IO type (i.e. collective vs. independent IO) at runtime. This means that we will provide an API for IO within this abstraction which allows the applications developer to write the application completely independent of the underlying IO subsystem.

\subsubsection{Shall support independent input / output.}

Independent IO - in this type of IO, a single file is created for each processor with the assumption that some other application will deal with the fragmented set of files. For example a parallel post processor might be written that understands the concept of the domain decomposition and contains a parallel rendering algorithm that just works on each fragment. Or, a harvester application could combine the fragments from each processor into a single file (not quite the same as concatenation because of duplicate nodes). The result of a harvester application would appear to the code user as if collective IO was performed.

\subsection{External Interface Requirements}

10.5.1 Shall support ExodusII / Nemesis file format. 


\section{Appendix A Requirements Not Met in this Release}

\section{A.1 Computational Environment}

\section{A.1.1 Warning/Error Handling}

A.1.1.1 Shall provide error termination facility that synchronizes all the application's parallel processes, outputs meta data, outputs specified bulk data, and performs "normal" termination the parallel processes.

The error termination capability will be available via direct invocation by the application or by invocation through a specified external signal.

\section{A.1.2 Coupling with non-SIERRA Application Object Code}

An application may require the integration of object code that is not developed using the SIERRA framework.

\section{A.1.2.1 Shall provide an interface to support external library input/output/ restart.}

\section{A.1.2.2 Shall identify external entities as "singletons" or "types."}

If an entity is a singleton then only one instance of that entity may exist in the application. If an entity is a type then multiple instances of that entity may exist in an application.

\section{A.1.3 Coupling with non-SIERRA Application Executable Code}

This is an open issue under discussion with the SIERRA user community. 


\section{A.2 Mesh (Discretization) Services}

\section{A.2.1 Mesh Objects}

A.2.1.1 Shall support particle method mesh object topologies and their mesh objects.

A.2.1.2 Shall support finite difference method mesh object topologies and their mesh objects.

\section{A.2.1.3 Shall support mesh object with general polyhedral topologies.}

\section{A.2.2 Subsets of Mesh Objects}

\section{A.2.2.1 Shall support specification of mutually exclusive active and inactive subsets of mesh objects.}

This functionality allows mesh objects to be input, marked and inactive, and then later "birthed" by moving them from the inactive to the active subset.

\section{A.2.2.2 Shall support geometry-correlated subsets of mesh objects.}

For example, a surface defines the physical extent over which boundary conditions, contact conditions, radiation heat transfer, etc. occur. This is a purely geometric concept. The initial implementations will no doubt be discretized in nature. However, the abstraction of a subset of mesh objects should support geometrical specifications for subsets.

\section{A.2.3 Dynamic Updates to Mesh Objects}

\section{A.2.3.1 Shall support deletion of "orphaned" mesh objects.}

Deletion of a set of higher dimensional mesh objects, e.g. elements, may result in one or more lower dimensional mesh objects, e.g. nodes, being detached from a mesh. These "orphaned" mesh objects should be detected and deleted.

\section{A.2.4 Dynamic Generation of "Intermediate" of FEM Mesh Objects}

Given a consistent subset of FEM mesh objects, for example the faces and nodes of a surface, an application may need the edges within that surface to perform a given computation. 
A.2.4.1 Given a consistent subset of FEM elements and nodes shall generate, upon request, the faces and/or edges of that subset of elements.

A.2.4.2 Given a consistent subset of FEM elements and nodes shall generate, upon request, the external faces of that subset of elements.

This requirement supports the contact mechanics need for "skinning a mesh" for its surface.
A.2.4.3 Given a consistent subset of FEM elements and nodes shall generate, upon request, the mid-edge nodes, mid-face nodes, or mid-element nodes.

\section{A.2.5 Hierarchical Refinement of Mesh Objects}

\section{A.2.5.1 Shall support refinement of any mesh object that can be partitioned into child mesh objects of the same topology.}

For example hexahedrals are partitioned into hexahedrals, quadrilaterals partitioned into quadrilaterals, or triangles partitioned into triangles.

\section{A.2.5.2 Shall support refinement of any mesh object that can be partitioned into child mesh objects of a topology consistent with the parent mesh object topology.}

For example, a pyramid may be partitioned into six child pyramids and four child tetrahedrals. This partitioning is consistent in that each triangular face is partitioned into four similar child triangles and the quadrilateral face is partitioned into four child quadrilaterals.

\section{A.2.5.3 Shall support specification of refinement-stencil for the refinement of mesh objects.}

The refinement-stencil defines how a mesh object and its topologically related mesh objects are to be refined. For example, a parent hexahedral is refined into eight similar hexahedrals, each quadrilateral face of the parent hexahedral is refined into four similar quadrilaterals, twelve interior quadrilaterals are generated, etc. 


\section{A.2.5.4 Shall support unrefinement of any parent mesh object that has been refined as long as the children of the parent mesh object are not refined.}

\section{A.2.5.5 Hierarchical refinement shall satisfy one-irregular neighbor constraint.}

SIERRA will always impose a two-to-one refinement rule for mesh objects. The two-to-one refinement rule constrains neighboring mesh objects, for example two quadrilaterals that share an edge, to have no more than one level of partitioning difference at their interface.

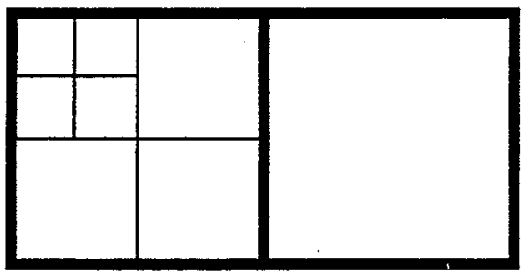

Conforms to the 2 tol constraint

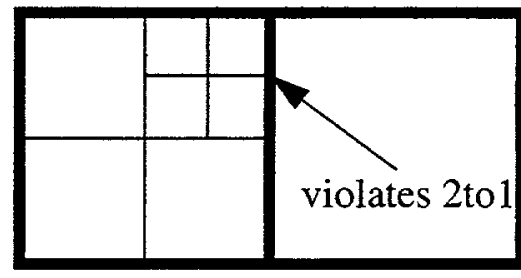

Violates the 2to1 constraint

\section{A.2.5.6 The child mesh objects created through hierarchical refinement shall} be initially placed into the same subsets as the parent mesh object.

\section{A.2.5.7 Should not support refinement of mesh objects that cannot be partitioned such that the two-to-one neighbor refinement constraint is achieved.}

\section{A.2.5.8 Shall provide inter-generational mesh object relations for hierarchical refinement.}

The parent mesh objects "know" their children and the child mesh objects "know" their parent.

\section{A.2.5.9 Shall support uniform hierarchical refinement of all mesh objects.}

The generation of a mesh object is defined by the number refinements that has occurred to produce the mesh object. For example, e.g. a generation zero or root mesh object is not the child of any other mesh object, a child of a root mesh object is generation one, a grandchild of a root mesh object is generation 2 , and so forth. 
A.2.5.10 Shall support computations on a given generation of mesh objects in a uniformly hierarchically refined mesh.

A.2.5.11 Shall support computations spanning multiple, specified, generations of a uniformly hierarchically refined mesh.

Examples of such computations include prolongation and restriction operators.

A.2.5.12 Shall support non-uniform hierarchical refinement of mesh objects.

A mesh object that has not been refined in a non-uniformly hierarchically refined mesh is referred to as a leaf mesh object.

A.2.5.13 Shall support computation on the leaf mesh objects of a nonuniformly hierarchically refined mesh.

\section{A.3 Variable (Field) Services}

\section{A.3.1 Variable Types}

A.3.1.1 Shall support predefined scalar, vector, and tensor types with complex-valued coefficients.

A.3.1.2 The SIERRA-intrinsic complex type shall be the aggregate of two SIERRA-intrinsic real values, a "Real" value and an "Imaginary" value.

A.3.1.3 Shall support application defined arrays of intrinsic complex values.

\section{A.4 Mechanics Algorithm Support Services}

\section{A.4.1 Master Element Services}

\section{A.4.1.1 Shall define an interface for Parent-Child Element Prolongation.}

This module defines the method interfaces for moving element state variables from a parent element to its children upon refinement under h-adaptivity. (not required until h-adaptivity is implemented). 


\section{A.4.1.2 Shall define an interface for Parent-Child Element Restriction.}

This module defines the method interfaces for moving the element state variables from the children elements to its parents upon refinement under h-adaptivity (not required until $\mathrm{h}$-adaptivity is implemented).

\section{A.4.1.3 Shall provide an initial implementation for parent-child prolongation and restriction algorithms.}

\section{A.5 Constraint Mechanics Services}

\section{A.5.0.1 Shall provide services to consistently handle and resolve a set of implicit constraints.}

\section{A.6 H-Adaptivity}

\section{A.6.0.1 Shall support h-adaptivity requests in a primary region to refine, unrefine, or leave a mesh object unchanged.}

The SIERRA Framework will not provide any error estimation mechanics capability. The determination of an h-adaptivity request is entirely within the mechanics developer's domain. SIERRA takes over after the error estimator/indicator has been computed and used to issue the request. SIERRA takes care of all the data movement required to create children from a parent (on divide) or coalesce children back into their parent (on undivide).

\section{A.6.0.2 Shall always honor h-adaptivity refinement requests.}

\section{A.6.0.3 Shall globally enforce a two-to-one refinement rule.}

SIERRA will always impose a two to one refinement rule. By this, we mean that two adjacent elements will differ at most by one generation of refinement. The unrefine request may not be honored because the two to one rule may require a propagation of the divided elements out into the mesh. It will be quite common that an element that has been previously divided due to the two to one refinement rule will be flagged for undivide because the error estimator indicates that the element is over-refined.

\section{A.6.0.4 Shall track the refinement conditions of refined mesh objects and mesh objects generated due to refinement.}

The refinement conditions denote at the least why a mesh object was refined, e.g. due to an explicit request or due to enforcement of the two-to-one refinement constraint. 


\section{A.6.0.5 Shall support the invocation of an application specified mechanics algorithm over the subset of newly-refined and/or newly-unrefined mesh objects.}

This algorithm may include special prolongation or restriction operators, or may perform algorithm specific initializations.

\section{A.6.0.6 Shall insure global consistency of mesh objects within a primary region.}

The SIERRA divide/undivide methods must automatically divide/undivide associated mesh objects, for example the faces and edges of a divided element.

\section{A.6.0.7 Shall reflect $h$-adaptivity modifications from the primary region to secondary regions.}

\section{A.7 Dynamic Parallel Decomposition / Load Balancing}

\section{A.7.1 Dynamic Load Balancing}

Dynamic load balancing is needed to achieve a scalable algorithm for any application which uses h-adaptivity. However, dynamic load balancing is also required for some applications which do not rely upon h-adaptivity. Consider an application which includes a complex constitutive model such as a concrete model. Under loads which produce no cracking, the constitutive model may be trivial whereas when cracks begin to form the computations may require a level of computation that is orders of magnitude more than the un-cracked case. Clearly, if some portion of the model is cracking and all of this portion is on a single processor, the computations will become extremely unbalanced. 
A.7.1.1 Shall support redistribution among processors of mesh objects within a primary or secondary region given a parallel partitioning specification.

A.7.1.2 Should support interrogation and communication of load measures.

A.7.1.3 Shall support assignment of load measures to mesh objects.

A.7.1.4 Shall support load balancing of mesh objects based upon load measures.

\section{A.8 Intra-Mechanics and Inter-Mechanics Transfer Services}

\section{A.8.1 Same-Mesh Transfers}

A.8.1.1 Shall support application defined control object for the transfer algorithm.

For example, the control object may be used for:

a. specifying tolerances in the geometric search step,

b. specifying parameters for steering the transfer projection/interpolation algorithm.

\section{A.8.2 Different Mesh Geometry based Transfers}

A.8.2.1 A general set of tolerances shall be provided to allow the user to control the behavior of the on-processor geometric search for cases such as where the geometric boundaries may not be exact due to discretization differences, roundoff, or deformations of the meshes.

A.8.2.2 Should support a geometric specification for the intersection of two greater-than-zero-dimensional mesh objects.

For example the area of overlap of two quadrilateral faces. 


\section{A.9 Linear Equation Solver Services}

A.9.1 Requirements for the FEI

A.9.1.1 Shall support direct solution of dense linear systems of equations.

A.9.1.2 Shall support queries for globally assembled matrix coefficients.

A.9.1.3 Shall support logical partitioning of the global linear system of equations.

A.9.1.4 Shall support solver operations with partitions of the global linear system of equations.

A.9.1.5 Shall support rectangular element matrix partial sum contributions.

A.9.1.6 Shall support rectangular element matrix partial sum contributions.

\section{A.10 Global Data Services}

\section{A.10.1 Output of Global Application Data}

A.10.1.1 Global application data describing the configuration of an application's mechanics algorithms shall be generated by the application upon request.

A.10.1.2 Non-constant global application data shall be output at every restart request.

A.10.2 Parsing of Input Leading to Global Application Data

A.10.2.1 Shall support verification of consistency of input with the application's mechanics algorithms.

\section{A.11 Bulk Data Input/Output Services}

Bulk data is the information associated with specific instances of mesh objects. Bulk data is often 
coupled to or dependent upon global application data; therefore, input/output of bulk data may be coupled to input/output of global application data.

\section{A.11:1 Multiple File Formats}

\section{A.11.1.1 Shall support collective input / output.}

Collective IO - in this type of IO, the application developer and code user has the concept of one output file for the entire model. The underlying IO system (in SIERRA, this is an IOController) is responsible for taking the contributions from each processor and combining them into a single file.

\section{A.11.2 External Interface Requirements}

\section{A.11.2.1 Shall support DMF file formats.}

\section{A.11.2.2 Shall support Parallel Data System / Parallel IO (PDS/PIO) file formats.}




\section{Disbribution:}

MS $0841 \quad$ P. J. Hommert, 9100

MS 0828 T. C. Bickel, 9101

MS $0828 \quad$ R. K. Thomas, 9104

MS $0826 \quad$ W. L. Hermina, 9111

MS $0834 \quad$ A. C. Ratzel, 9112

MS 0835 S. N. Kempka, 9113

MS 0827 R. O. Griffith, 9114

MS $0836 \quad$ L. M. Taylor, 9121 (5)

MS $0836 \quad$ H. C. Edwards , 9121 (10)

MS $0836 \quad$ J. R. Stewart, 9121 (10)

MS 0827 J. D. Zepper, 9136

MS $0835 \quad$ J. S. Peery, 9231

MS $9217 \quad$ R. L. Clay, 8980

MS 9018 Central Technical Files, 8940-2

MS 0899 Technical Library, 4916 (2)

MS 0619 Review and Approval Desk, 4912

David Cookerly

TRW Systems \& Information Technolgy

4243 Piedras Drive East, Suit 100

San Antonio, TX 78228

Joe P. Holland

Kelly Space \& Technology

294 South Leland Norton Way, Suit 3

San Bernadino, CA 92408

Tom Zebrowski

Knowls Atomic Power Laboratory, Bldg M9

P.O. Box 1072

Schenectady, NY 12301-1072

High Performance Computing Modernization Office 1010 North Glebe Road, Suite 510

ATTN: John Grosh

Arlington, VA 22201 